\title{
Tests of a density-based local pseudopotential for sixteen simple metals
}

\author{
L. Pollack, J. P. Perdew, and J. He \\ Department of Physics and Quantum Theory Group, Tulane University, New Orleans, Louisiana 70118 \\ M. Marques, F. Nogueira, and C. Fiolhais \\ Department of Physics, University of Coimbra, P3000 Coimbra, Portugal
}

(Received 11 December 1996)

\begin{abstract}
A comprehensive study of the lattice dynamics, elastic moduli, and liquid metal resistivities for 16 simple metals in the bcc and fcc crystal structures is made using a density-based local pseudopotential. The phonon frequencies exhibit excellent agreement with both experiment and nonlocal pseudopotential theory. The bulk modulus is evaluated by the long wave and homogeneous deformation methods, which agree after a correction is applied to the former. Calculated bulk and Voigt shear moduli are insensitive to crystal structure, and long-wavelength soft modes are found in certain cases. Resistivity calculations confirm that electrons scatter off the whole Kohn-Sham potential, including its exchange-correlation part as well as its Hartree part. All of these results are found in second-order pseudopotential perturbation theory. However, the effect of a nonperturbative treatment on the calculated lattice constant is not negligible, showing that higher-order contributions have been subsumed into the pseudopotential by construction. For bcc sodium, the band structures of local and nonlocal pseudopotentials are found to be almost identical. [S0163-1829(97)01321-0]
\end{abstract}

\section{INTRODUCTION AND SUMMARY}

The pseudopotential formalism, ${ }^{1,2}$ in which a weak effective interaction is set up between atomic cores and valence electrons, does an unexpectedly good job of reproducing properties of close-packed metals in second-order perturbation theory. ${ }^{3}$ A recently developed local pseudopotential for simple metals ${ }^{4}$ has been constructed specifically to be used in second-order ground-state energy calculations. This local pseudopotential, which we shall call density based, is tested here in a systematic study on a large class of metals.

To understand the spirit behind this density-based formalism, it is helpful to compare two different pseudopotential constructions. Conventional norm-conserving 5 pseudopotentials are constructed for a free-atom reference state, in which the nonlocal pseudopotential $w\left(\boldsymbol{r}, \boldsymbol{r}^{\prime}\right)$ reproduces low-lying valence-electron Kohn-Sham orbitals $\Psi_{i}(\mathbf{r})$ outside the core. Our density-based pseudopotential is constructed for a crystalline reference state, in which the local pseudopotential $w(r)$ reproduces key features of the electron density $\rho(\mathbf{r})$ outside the core. More precisely (in atomic units with $e^{2}=\hbar=m=1$ )

$$
w(r)=\frac{-z}{r}+w_{\mathrm{R}}(\alpha, R ; r),
$$

where $z$ is the valence. The core repulsion $w_{\mathrm{R}}$ depends analytically upon $r$, the distance from the electron to the center of the ion, and decays like $e^{-r / R}$ as $r \rightarrow \infty$. The two parameters $\alpha$ and $R$ reproduce the all-electron interstitial density and the observed equilibrium lattice constant, where the density $\rho(\mathbf{r})$ is computed to first order and the total energy $E$ to second order in the pseudopotential, using the local density approximation (LDA) for exchange and correlation. The parameter $R$ turns out to be essentially the decay length of the highest-occupied core orbital of $s$ or $p$ symmetry.
In previous work ${ }^{4}$ this local pseudopotential was shown to give a good account of crystal structure, bulk binding energy, and bulk modulus for the simple metals. Advantages of locality include computational simplicity, physical transparency, and suitability for use with density-functional theory (which in principle requires a local external potential). A disadvantage is that local pseudopotentials are less transferable $^{6}$ to nonreference environments than nonlocal ones.

Comprehensive unified studies of several metals with pseudopotentials are surprisingly rare, even though the simple metals justify use of a single, simple model to describe them. Rose and Shore ${ }^{7}$ delineated trends in the elastic constants for both simple and transition metals, stressing the importance of interstitial density. Moriarty ${ }^{8}$ calculated lattice dynamics, cohesive energies, and liquid metal resistivities using a nonlocal pseudopotential. Our work entails not only the lattice dynamics, but also the elastic moduli and liquid metal resistivities for 16 metals $(\mathrm{Li}, \mathrm{Na}, \mathrm{K}, \mathrm{Rb}, \mathrm{Cs}, \mathrm{Ba}, \mathrm{Be}$, $\mathrm{Mg}, \mathrm{Ca}, \mathrm{Sr}, \mathrm{Al}, \mathrm{Ga}, \mathrm{In}, \mathrm{Tl}, \mathrm{Sn}, \mathrm{Pb})$.

The first root of our interest in the calculation of phonon frequencies and elastic constants goes back to the work of Rasolt and Taylor. ${ }^{9,10}$ They constructed nonlocal pseudopotentials which, in first-order perturbation theory, reproduce the electron density around an ion in a uniform electron gas. Perdew and Vosko ${ }^{11}$ showed that the same procedure could be applied to construct local pseudopotentials, which yield realistic phonon frequencies to second order for $\mathrm{Li}$, where the pseudopotential is expected to be both nonlocal and strong; similar calculations have been made for Al (Refs. 12-14) and $\mathrm{Mg} .{ }^{15}$ In our present study, although we no longer invoke an ion in an electron gas, we still follow the primary directive of this earlier work: make the density outside the core correct to first-order in order to make the total energy correct in a second-order calculation. 
The second root of our interest lies in the systematic calculations of simple-metal elastic constants by Ling and Gelatt. ${ }^{16}$ They used a local pseudopotential constructed from the bulk stability condition alone to identify chemical trends in the bulk and shear moduli for 19 metals. The local pseudopotential we use here is more realistic ${ }^{4}$ in its description of band-structure (second-order) effects, but still recognizes the key role played by the bulk equilibrium or zeropressure condition. Except for the metals $\mathrm{Be}, \mathrm{Ca}, \mathrm{Sr}$, and $\mathrm{Ba}$, where pseudopotential nonlocality can be especially important, our calculated phonon frequencies and elastic constants are in satisfactory agreement with experiment.

While phonon frequencies and elastic constants can be related directly to the total energy, the electrical resistivity cannot. Thus, we do not expect and do not find that our local pseudopotential is as accurate for the latter as for the former. Nevertheless, our calculated resistivities are sufficiently realistic to discriminate between proper and improper screening of the electron-ion interaction, as we show later in this article.

Since our local pseudopotential is constructed in and for second-order perturbation theory, it is not necessarily optimal for a nonperturbative treatment, as we show by calculating lattice constants both ways with the same pseudopotential. However, its two principles of construction (matching to both the all-electron interstitial density and the observed lattice constant) can also be used to find new parameters $\alpha$ and $R$ in Eq. (1) that are appropriate for a nonperturbative treatment. We have done this for sodium, and find that the resulting band structure is almost identical to that of a normconserving nonlocal pseudopotential. This nonperturbative approach should also permit the extension of our local pseudopotential to a broader class of materials, including $\mathrm{Si}$ and Ge.

\section{LATTICE DYNAMICS}

To obtain phonon frequencies for cubic crystals, we use the diagonal form ${ }^{11}$ of the dynamical matrix for phonon wave vectors $\mathbf{q}$ pointing in the three high-symmetry directions:

$$
\omega^{2}(\boldsymbol{q}, \hat{\boldsymbol{\epsilon}})=\omega_{d}^{2}(\boldsymbol{q}, \hat{\boldsymbol{\epsilon}})-\omega_{e}^{2}(\boldsymbol{q}, \hat{\boldsymbol{\epsilon}})
$$

where

$$
\begin{aligned}
& \omega_{d}^{2}(\boldsymbol{q}, \hat{\boldsymbol{\epsilon}})= \omega_{p}^{2}\left[\frac{(\hat{\boldsymbol{\epsilon}} \cdot \boldsymbol{q})^{2}}{q^{2}}+\sum_{\boldsymbol{G} \neq 0}\left(\frac{[\hat{\boldsymbol{\epsilon}} \cdot(\boldsymbol{q}+\boldsymbol{G})]^{2}}{(\boldsymbol{q}+\boldsymbol{G})^{2}} e^{-(\boldsymbol{q}+\boldsymbol{G})^{2} / 4 \eta}\right.\right. \\
&\left.\left.-\frac{[\hat{\boldsymbol{\epsilon}} \cdot \boldsymbol{G}]^{2}}{G^{2}} e^{-G^{2} / 4 \eta}\right)\right], \\
& \omega_{e}^{2}(\boldsymbol{q}, \hat{\boldsymbol{\epsilon}})= \omega_{p}^{2}\left[\frac{(\hat{\boldsymbol{\epsilon}} \cdot \boldsymbol{q})^{2}}{q^{2}} \Phi(\boldsymbol{q})+\sum_{\boldsymbol{G} \neq 0}\left(\frac{[\hat{\boldsymbol{\epsilon}} \cdot(\boldsymbol{q}+\boldsymbol{G})]^{2}}{(\boldsymbol{q}+\boldsymbol{G})^{2}} \Phi(\boldsymbol{q}+\boldsymbol{G})\right.\right. \\
&\left.\left.-\frac{[\hat{\boldsymbol{\epsilon}} \cdot \boldsymbol{G}]^{2}}{G^{2}} \Phi(\boldsymbol{G})\right)\right], \\
& \Phi(Q)=\frac{-\chi_{0}(Q)}{\left(k_{F} / \pi^{2}\right)} \frac{w(Q)}{\left(k_{F}^{2} / 3\right) \boldsymbol{\epsilon}(Q)} \frac{w(Q)}{4 \pi z / \Omega_{0} Q^{2}},
\end{aligned}
$$

and

$$
\omega_{p}^{2}=4 \pi z^{2} /\left(\Omega_{0} M\right)
$$

where $\omega_{p}$ is the plasma frequency, $\Omega_{0}=z 4 \pi r_{s}^{3} / 3$ is the equilibrium volume per ion, and $M$ is the atomic mass. The polarization is $\hat{\boldsymbol{\epsilon}}$ and $\mathbf{G}$ represents a reciprocal-lattice vector. Analytic expressions for the noninteracting linear-response function $\chi_{0}(Q)$, the dielectric function $\epsilon(Q)$, and the Fourier transform of the pseudopotential $w(Q)$, can be found in Ref. 4. The convergence parameter $\eta \rightarrow \infty$ is defined below.

The interpretation of Eq. (2) is straightforward. The first term, $\omega_{d}^{2}$, accounts for the motion of positive point ions embedded in a rigid, uniform negative background. The next term, $\omega_{e}^{2}$, accounts for electrons which are no longer rigid, screening the effects of the moving ions. Reasonable results for $\omega_{d}^{2}$ are impossible to obtain directly since the singularity of the Coulomb potential prevents the convergence of sums over reciprocal-lattice vectors. The solution ${ }^{1}$ is to multiply each summation by the decaying exponential shown in Eq. (3), sometimes called a Gaussian compensating cap on the ion, in order to make the series converge. The parameter that controls convergence is $\eta$, and we let $1 / \eta$ go to zero at the end.

We studied each of the metals in both the body-centeredcubic (bcc) and face-centered-cubic (fcc) crystal structures, obtaining phonon frequencies for all 32 cases. In Table I, calculated phonon frequencies on the Brillouin-zone boundary are compared to experiment, where available, with a root-mean-square (RMS) relative error of $19 \%$. We find that our local pseudopotential yields comparable accuracy to the nonlocal one of Moriarty ${ }^{8}$ (also applied to second-order), for which the RMS relative error is $21 \%$. Calculated frequencies for $\mathrm{Li}, \mathrm{Al}$, and $\mathrm{Pb}$ are too high, and for $\mathrm{Li}$ we do not achieve the crossover of the transverse $(T)$ and longitudinal $(L)$ branches in the [100] direction, an effect discussed by Sen $e t$ $a l .{ }^{17}$ The alkali metals, excluding $\mathrm{Li}$, show excellent agreement with experiment.

\section{ELASTIC MODULI AND SOFT MODES}

Cubic crystals have three independent elastic constants $^{18,19}\left(C_{11}, C_{12}\right.$, and $\left.C_{44}\right)$; they determine the bulk modulus or incompressibility under hydrostatic pressure

$$
B=\left(C_{11}+2 C_{12}\right) / 3,
$$

as well as the two shear moduli for volume-conserving processes,

$$
C^{\prime}=\left(C_{11}-C_{12}\right) / 2,
$$

and $C_{44}$, which measure rigidity against tetragonal and angle-bending distortions of the unit cube, respectively. The Voigt or directionally averaged shear modulus ${ }^{16}$

$$
\mu=\left(2 C^{\prime}+3 C_{44}\right) / 5
$$

is also $\mu_{p}$, the single shear modulus of a polycrystalline sample, provided ${ }^{18}$ that the cubic single crystal is fairly isotropic $\left(C^{\prime} \approx \mu\right)$. Similar formulas for hexagonal closepacked crystals are Eq. (3.6) of Ref. 20 and Eq. (22) of Ref. 16. 
TABLE I. Calculated phonon frequencies $(\mathrm{THz})$ on the Brillouin-zone boundary. Experimental values are found in Ref. 8, except Cs (Ref. 53), Ba (Ref. 54), Ca (Ref. 55), and Sr (Ref. 56).

\begin{tabular}{|c|c|c|c|c|c|c|c|c|}
\hline \multirow{3}{*}{$\frac{b c c}{\mathrm{Li}}$} & Theor. & \multirow{2}{*}{ Expt. } & Theor. & \multirow{2}{*}{ Expt. } & Theor. & \multirow{2}{*}{ Expt. } & Theor. & \multirow{2}{*}{$\begin{array}{l}\text { Expt. } \\
\text { ] }\end{array}$} \\
\hline & $L[100]$ & & $L[110]$ & & $T 1[110]$ & & $T 2[110]$ & \\
\hline & 9.09 & 8.82 & 10.34 & 9.00 & 2.10 & 1.90 & 6.19 & 5.70 \\
\hline $\mathrm{Na}$ & 3.63 & 3.58 & 4.06 & 3.82 & 0.84 & 0.93 & 2.51 & 2.56 \\
\hline K & 2.15 & 2.21 & 2.42 & 2.40 & 0.52 & 0.55 & 1.46 & 1.50 \\
\hline $\mathrm{Rb}$ & 1.30 & 1.39 & 1.45 & 1.50 & 0.31 & 0.34 & 0.89 & 0.96 \\
\hline Cs & 0.93 & 0.90 & 1.04 & 1.07 & 0.23 & 0.22 & 0.64 & 0.58 \\
\hline $\mathrm{Ba}$ & 2.04 & 2.15 & 2.27 & 2.30 & 0.13 & 0.75 & 1.49 & 1.53 \\
\hline fcc & \multicolumn{2}{|c|}{$L[100]$} & \multicolumn{2}{|c|}{$T[100]$} & \multicolumn{2}{|c|}{$L[111]$} & \multicolumn{2}{|c|}{$T[111]$} \\
\hline $\mathrm{Be}$ & 22.15 & & 14.25 & & 22.83 & & 9.38 & \\
\hline $\mathrm{Mg}$ & 8.24 & & 5.53 & & 8.34 & & 3.62 & \\
\hline $\mathrm{Ca}$ & 5.10 & 4.52 & 3.46 & 3.63 & 5.15 & 4.61 & 2.24 & 2.36 \\
\hline $\mathrm{Sr}$ & 3.05 & 3.20 & 2.07 & 2.20 & 3.07 & 3.00 & 1.34 & 1.40 \\
\hline $\mathrm{Al}$ & 11.28 & 9.67 & 6.81 & 5.81 & 11.59 & 9.64 & 4.59 & 4.18 \\
\hline $\mathrm{Ga}$ & 4.38 & & 2.63 & & 4.57 & & 1.72 & \\
\hline In & 3.30 & & 2.03 & & 3.42 & & 1.36 & \\
\hline $\mathrm{Tl}$ & 2.05 & & 1.27 & & 2.13 & & 0.82 & \\
\hline $\mathrm{Sn}$ & 3.52 & & 2.16 & & 3.77 & & 1.39 & \\
\hline $\mathrm{Pb}$ & 2.40 & 1.86 & 1.48 & 0.89 & 2.52 & 2.19 & 0.93 & 0.89 \\
\hline
\end{tabular}

In a polycrystalline (macroscopically isotropic) sample, Young's (stretch) modulus $Y$ and Poisson's (strain) ratio $\sigma$ $\operatorname{are}^{21}$

$$
\begin{gathered}
Y=\frac{9 B}{1+3 B / \mu_{p}}, \\
\sigma=\frac{1}{2}\left(\frac{3 B}{\mu_{p}}-2\right) /\left(\frac{3 B}{\mu_{p}}+1\right) .
\end{gathered}
$$

The fractional volume change upon stretching is $\delta V / V=(1-2 \sigma) \delta \ell / \ell$, where $\delta \ell$ is the increase in length $\ell$. Two interesting limits are the isotropic Cauchy solid $\left(\mu_{p} / B=3 / 5\right.$ whence $Y=3 B / 2$ and $\left.\sigma=1 / 4\right)$ and the liquid $\left(\mu_{p} / B=0\right.$ whence $Y=0$ and $\left.\sigma=1 / 2\right)$. Of course the Cauchy relation $C_{44}=C_{12}$ is true only for a model of pair potentials in equilibrium, not for real metals. ${ }^{22}$

The elastic constants can be found from the phonon frequencies $\omega(\mathbf{q})$ and their sound velocities ${ }^{23} \partial \omega / \partial q$ by the $q \rightarrow 0$ method $^{24}$ of long waves (LW). The bulk modulus determined in this way from Eq. (7), $B_{\mathrm{LW}}$, is in principle equivalent to that found by the method of homogeneous deformation (HD),

$$
B=B_{\mathrm{HD}}=V\left(\partial^{2} E / \partial V^{2}\right)_{N},
$$

where $E$ is the total energy and $V$ is the volume of the crystal. However, the numerical results $B_{\mathrm{LW}}$ and $B_{\mathrm{HD}}$ differ ${ }^{25-27}$ when all expressions are truncated at second order in the electron-ion interaction; $B_{\mathrm{HD}}$ is more realistic because it includes a change in the dielectric function due to a change in the volume per ion, ${ }^{17}$ which is missing from $B_{\mathrm{LW}}$.

Using our local pseudopotential, we evaluated $B_{\mathrm{LW}}$, $B_{\mathrm{HD}}$, and an independent expression ${ }^{25,28,29}$ for their difference $\Delta$. Table II compares these results. We indeed find
$\Delta \approx\left(B_{\mathrm{HD}}-B_{\mathrm{LW}}\right)$, except in some of the higher-valence metals for which our LW result is probably somewhat in error due to the delicate cancellation between ionic and electron contributions to the phonon frequencies. Adding $\Delta$ to $B_{\mathrm{LW}}$ also brings the calculated LW bulk modulus much closer to experiment, with a root-mean-square relative error of only $11 \%$. Jones ${ }^{27}$ argued that $\Delta /\left(B_{\mathrm{LW}}+\Delta\right)$ should not be large for the alkalis (we find it is roughly 30\%), but might be significant for the polyvalents (where we find that it varies from $2 \%$ for $\mathrm{Ga}$ to $107 \%$ for $\mathrm{Be}$ ).

As shown in Table II, the bulk modulus is insensitive to the difference between the bcc and fcc structures. This result is not unexpected, since Wigner and Seitz ${ }^{30,31}$ successfully calculated bulk moduli from a model in which the polyhedral Wigner-Seitz cell was replaced with a sphere of the same volume. Table II also shows our calculated long-wave shear moduli $C^{\prime}$ and $\mu$, which require no correction since they describe volume-conserving distortions. As anticipated by Ling and Gelatt, ${ }^{16} \mu$ turns out to be relatively insensitive to the difference between bcc and fcc structures; however, $C^{\prime}$ is quite sensitive to crystal structure. In view of the substantial differences between $C^{\prime}$ and $\mu$, with $C^{\prime} \ll \mu$, we have tried to report only single-crystal (and not polycrystalline) experimental values for comparison with the results of our calculations.

Our result $C^{\prime}<0$ means that the cubic structure is unstable against tetragonal distortion, under which bcc can be transformed continuously to fcc. ${ }^{32,33} \mathrm{We}$ find this instability in the bcc structure of all our metals with $z \geqslant 2$, consistent with the finding of Fiolhais et al. ${ }^{4}$ that fcc has a lower calculated total energy than bcc for these metals. Additionally, our $C^{\prime}<0$ result is always associated with long-wavelength phonon soft modes, ${ }^{8}$ which for bcc occur in the $T 1$ [110] branch. For $\mathrm{Ca}, \mathrm{Sr}$, and $\mathrm{Ba}$ we also find $C^{\prime}<0$ for the fcc 
TABLE II. The bulk modulus, $B\left(=B_{\mathrm{LW}}+\Delta\right)$, and the shear moduli $\mu$ and $C^{\prime}$; values $\left(10^{10} \mathrm{dyn} / \mathrm{cm}^{2}\right)$ have been calculated for bcc and fcc crystal structures. Experimental numbers are for the observed structures, which are fcc or bcc as shown in the first line of each metal, except for Be (hcp), $\mathrm{Mg}$ (hcp), In (tetragonal for $B$, hcp for $\mu$ ), $\mathrm{Tl}$ (hcp) and Sn (tetragonal for $B$ ). All experimental numbers are taken from Ref. 57 unless noted. Experimental numbers in parentheses indicate polycrystalline data taken from Ref. 21; experimental numbers for $\mathrm{Ca}, \mathrm{Sr}$, and $\mathrm{Ba}$ are from Refs. 55, 56, and 54, respectively.

\begin{tabular}{|c|c|c|c|c|c|c|c|c|c|}
\hline & & \multirow[b]{2}{*}{$\Delta$} & \multirow[b]{2}{*}{$B_{\mathrm{HD}}-B_{\mathrm{LW}}$} & \multicolumn{2}{|c|}{$B$} & \multicolumn{2}{|c|}{$\mu$} & \multicolumn{2}{|c|}{$C^{\prime}$} \\
\hline & & & & Theor. & Expt. & Theor. & Expt. & Theor. & Expt. \\
\hline \multirow[t]{2}{*}{$\mathrm{Li}$} & bcc & -8.3 & -8.2 & 13.8 & 13.3 & 8.1 & 6.9 & 1.5 & 1.2 \\
\hline & fcc & -8.3 & -8.3 & 14.0 & & 8.1 & & 1.7 & \\
\hline \multirow[t]{2}{*}{$\mathrm{Na}$} & bcc & 2.3 & -2.2 & 7.0 & 7.3 & 3.7 & 3.7 & 0.6 & 0.7 \\
\hline & fcc & -2.2 & -2.3 & 7.1 & & 3.6 & & 0.8 & \\
\hline \multirow[t]{2}{*}{ K } & bcc & -1.1 & -1.1 & 3.4 & 3.7 & 1.7 & 1.9 & 0.3 & 0.4 \\
\hline & fcc & -1.1 & -1.1 & 3.4 & & 1.7 & & 0.3 & \\
\hline \multirow[t]{2}{*}{$\mathrm{Rb}$} & bcc & -0.7 & -0.7 & 2.6 & 3.1 & 1.3 & 1.4 & 0.2 & 0.3 \\
\hline & fcc & -0.7 & -0.7 & 2.6 & & 1.3 & & 0.2 & \\
\hline \multirow[t]{2}{*}{ Cs } & bcc & -0.5 & -0.5 & 2.0 & 2.3 & 1.0 & 1.0 & 0.2 & 0.2 \\
\hline & fcc & -0.5 & -0.5 & 2.0 & & 0.9 & & 0.2 & \\
\hline \multirow[t]{2}{*}{$\mathrm{Ba}$} & bcc & -3.1 & -3.1 & 10.4 & 9.4 & 5.5 & 7.8 & -0.1 & 4.2 \\
\hline & fcc & -1.9 & -2.1 & 11.0 & & 4.6 & & -0.6 & \\
\hline \multirow[t]{2}{*}{$\mathrm{Be}$} & fcc & -100.1 & -103.1 & 93.5 & 115.6 & 52.3 & 153.1 & 4.6 & \\
\hline & bcc & -104.1 & -104.3 & 84.9 & & 55.9 & & -10.2 & \\
\hline \multirow[t]{2}{*}{$\mathrm{Mg}$} & fcc & -11.1 & -11.9 & 32.7 & 36.9 & 15.3 & 19.4 & 0.6 & \\
\hline & bcc & -13.4 & -13.5 & 30.5 & & 17.1 & & -1.7 & \\
\hline \multirow[t]{2}{*}{$\mathrm{Ca}$} & fcc & -4.1 & -4.5 & 17.0 & 21.4 & 7.5 & 11.7 & -1.5 & 4.8 \\
\hline & bcc & -6.5 & -6.5 & 15.9 & & 9.2 & & -0.2 & \\
\hline \multirow[t]{2}{*}{$\mathrm{Sr}$} & fcc & -2.6 & -2.8 & 12.5 & 12.0 & 5.4 & 8.3 & -1.1 & 2.7 \\
\hline & bcc & -4.3 & -4.3 & 11.7 & & 6.7 & & -0.1 & \\
\hline \multirow[t]{2}{*}{$\mathrm{Al}$} & fcc & -40.1 & -43.9 & 79.1 & 79.4 & 38.5 & 29.0 & 21.6 & 26.1 \\
\hline & bcc & -33.2 & -34.7 & 79.2 & & 33.0 & & -16.8 & \\
\hline \multirow[t]{2}{*}{$\mathrm{Ga}$} & fcc & 1.1 & -2.9 & 51.6 & $(56.8)$ & 13.8 & $(37.4)$ & 5.7 & \\
\hline & bcc & 0.3 & -1.8 & 51.2 & & 13.8 & & -4.6 & \\
\hline \multirow[t]{2}{*}{ In } & fcc & -0.6 & -3.2 & 39.0 & 41.6 & 12.5 & 6.7 & 6.1 & \\
\hline & bcc & -0.3 & -1.7 & 38.6 & & 11.8 & & -4.6 & \\
\hline \multirow[t]{2}{*}{$\mathrm{Tl}$} & fcc & 4.6 & 2.0 & 33.0 & 38.2 & 8.2 & 7.6 & 3.4 & \\
\hline & bcc & 3.9 & 2.5 & 32.4 & & 8.4 & & -1.8 & \\
\hline \multirow[t]{2}{*}{$\mathrm{Sn}$} & fcc & -1.4 & -6.1 & 53.1 & 57.9 & 14.1 & $(20.4)$ & 4.7 & \\
\hline & bcc & 0.2 & -2.3 & 52.2 & & 12.3 & & -11.5 & \\
\hline \multirow[t]{2}{*}{$\mathrm{Pb}$} & fcc & 2.6 & -1.6 & 44.7 & 48.8 & 10.8 & 13.7 & 3.4 & 5.1 \\
\hline & bcc & 1.7 & -0.6 & 43.7 & & 11.1 & & -3.1 & \\
\hline
\end{tabular}

structure, consistent with the finding of Fiolhais $e t$ al. ${ }^{4}$ that the hexagonal close-packed structure (hcp) has the lowest calculated total energy for these metals. This result is of course a failure of pseudopotential locality, ${ }^{8,34}$ since $\mathrm{Ca}$ and $\mathrm{Sr}$ are really fcc while $\mathrm{Ba}$ is really bcc.

As shown in Table III, all our bcc phonon soft modes disappear under pressure when the volume $\Omega$ per ion is reduced far enough. This is consistent with the observation ${ }^{35}$ that $\mathrm{Mg}$ transforms to the bcc structure under pressure. For $\mathrm{Ca}, \mathrm{Sr}$, and $\mathrm{Ba}$, the calculated bcc soft modes disappear under very slight compression. Recently Milstein and Rasky ${ }^{36}$ have shown that the bcc structure of the alkalis can go unstable under pressure, with $C^{\prime}<0$.

\section{RESISTIVITY}

As a more sensitive test of our density-based local pseudopotential, we have evaluated the electrical resistivities
TABLE III. Fraction of the equilibrium volume $\left(\Omega_{0}\right)$ at which the bcc phonon soft modes of the $T 1$ [110] branch disappear.

\begin{tabular}{lc}
\hline \hline Metal & $\Omega / \Omega_{0}$ \\
\hline $\mathrm{Be}$ & 0.75 \\
$\mathrm{Mg}$ & 0.87 \\
$\mathrm{Al}$ & 0.25 \\
$\mathrm{Ga}$ & 0.28 \\
$\mathrm{In}$ & 0.35 \\
$\mathrm{Tl}$ & 0.41 \\
$\mathrm{Sn}$ & 0.06 \\
$\mathrm{~Pb}$ & 0.09 \\
$\mathrm{Ca}$ & 0.97 \\
$\mathrm{Sr}$ & 0.98 \\
$\mathrm{Ba}$ & 0.97 \\
\hline \hline
\end{tabular}


TABLE IV. Liquid metal electrical resistivity $\rho$ (in $\mu \Omega \mathrm{cm}$ ) at the melting point. The resistivity $\rho$ has been calculated for the observed valence $z$ and density parameter $r_{s}$, using our local pseudopotential and three different choices of dielectric function, as described in the text; the most correct choice is LDA/LDA. Lacking an experimental value for $\mathrm{Be}$, we have listed the value calculated from the nonlocal pseudopotential in Ref. 8. The atomic unit of resistivity is $21.7 \mu \Omega \mathrm{cm}$.

\begin{tabular}{|c|c|c|c|c|c|c|}
\hline & \multirow[b]{2}{*}{$z$} & \multirow[b]{2}{*}{$r_{s}^{\mathrm{a}}$} & \multicolumn{4}{|c|}{$\rho$} \\
\hline & & & Har/Har & Har/LDA & LDA/LDA & Expt. $^{a}$ \\
\hline $\mathrm{Li}$ & 1 & 3.33 & 4 & 2 & 7 & 25 \\
\hline $\mathrm{Na}$ & 1 & 4.05 & 7 & 2 & 12 & 10 \\
\hline K & 1 & 5.01 & 7 & 1 & 14 & 13 \\
\hline $\mathrm{Rb}$ & 1 & 5.31 & 8 & 1 & 15 & 22 \\
\hline Cs & 1 & 5.83 & 9 & 0 & 20 & 36 \\
\hline $\mathrm{Be}$ & 2 & 1.94 & 6 & 4 & 8 & (77) \\
\hline $\mathrm{Mg}$ & 2 & 2.74 & 10 & 6 & 15 & 27 \\
\hline $\mathrm{Ca}$ & 2 & $3.40^{\mathrm{b}}$ & 16 & 9 & 27 & $23^{\mathrm{b}}$ \\
\hline $\mathrm{Sr}$ & 2 & $3.70^{\mathrm{b}}$ & 18 & 10 & 33 & $87^{\mathrm{b}}$ \\
\hline $\mathrm{Ba}$ & 2 & 3.83 & 18 & 9 & 33 & 134 \\
\hline $\mathrm{Al}$ & 3 & 2.16 & 12 & 9 & 17 & 24 \\
\hline $\mathrm{Ga}$ & 3 & 2.17 & 16 & 11 & 23 & 26 \\
\hline In & 3 & 2.44 & 16 & 10 & 25 & 33 \\
\hline $\mathrm{Tl}$ & 3 & 2.51 & 29 & 18 & 46 & 73 \\
\hline Sn & 4 & 2.27 & 23 & 15 & 34 & 48 \\
\hline $\mathrm{Pb}$ & 4 & 2.37 & 34 & 22 & 52 & 95 \\
\hline
\end{tabular}

${ }^{\mathrm{a}}$ Reference 38 .

${ }^{\mathrm{b}}$ Reference 40 .

of the liquid metals from the Ziman formula ${ }^{37-39}$

$$
\rho=\frac{4 \pi}{z k_{F}^{3}} \int_{0}^{2 k_{F}} \frac{d Q}{Q} S(Q)\left[\frac{w(Q)}{\left(4 \pi / Q^{2}\right) \epsilon(Q)}\right]^{2} .
$$

Here $S(Q)$ is the ion-ion structure factor, for which we use the hard-sphere model of Ref. 38, with packing fraction 0.45 .

Table IV compares theory and experiment ${ }^{38,40}$ at the melting point, and shows the experimental density parameter $r_{s}$ for that temperature. When the dielectric function $\epsilon(Q)$ is properly treated in the local density approximation $\left(\rho_{\text {LDA/LDA }}\right.$ in Table IV), reasonable agreement with experiment is found for most of the simple metals, but not for those in which the pseudopotential should be strongly nonlocal $(\mathrm{Li}$, $\mathrm{Be}$, and the alkaline earths). For the latter metals, the nonlocal pseudopotential calculation of Moriarty ${ }^{8}$ gives significantly more realistic resistivities.

The Ziman formula, Eq. (13), is highly sensitive ${ }^{41}$ to all of its inputs $S(Q), w(Q)$, and $\epsilon(Q)$. The correct dielectric function $\epsilon(Q)$ is ${ }^{42}$ the same one that appears in our calculation of phonon frequencies and total energies: Eq. (3.7) of Ref. 4, which corresponds to electrons scattering off fluctuations of the self-consistent Kohn-Sham potential in the local density approximation (LDA), with the electron-density response also evaluated in LDA. The results of this treatment are labeled $\rho_{\text {LDA/LDA }}$ in Table IV, and are virtually identical to resistivities that we calculated with the "exact" (beyond LDA) local field factor. ${ }^{43}$
TABLE V. Equilibrium lattice constants (bohr) for three simple metals. For bcc sodium and fcc aluminium, the lattice constant is the cube side. For hcp magnesium, it is the hexagon radius $a$, where the ratio $c / a$ is fixed at its experimental value 1.625 . Each theoretical lattice constant was found by minimizing the total energy in a nonperturbative calculation. The local pseudopotential is the one used in our perturbative calculation of phonon frequencies and elastic constants, while the nonlocal ( $s p d$ ) one is Hamann's (Ref. 48). Results for the local $s$-electron part of Hamann's potential are also shown.

\begin{tabular}{ccccc}
\hline \hline Metal & $\begin{array}{c}\text { Local } \\
\text { (unrevised) }\end{array}$ & $\begin{array}{c}\text { Hamann } \\
s\end{array}$ & $\begin{array}{c}\text { Hamann } \\
s p d\end{array}$ & Expt. \\
\hline $\mathrm{Na}$ & 8.11 & 7.42 & 7.50 & 7.98 \\
$\mathrm{Mg}$ & 6.38 & 5.94 & 5.90 & 6.05 \\
$\mathrm{Al}$ & 8.48 & 7.88 & 7.48 & 7.64 \\
\hline \hline
\end{tabular}

Other choices for $\epsilon(Q)$, corresponding to other positionings of the local field factor $G_{\mathrm{XC}}(Q)$ in Eq. (3.7) of Ref. 4, are sometimes encountered in the literature, but are expected to yield less satisfactory resistivities, as shown in Table IV. Neglect of the local field factor, i.e., use of the Lindhard ${ }^{41}$ $\epsilon(Q)$, corresponds to electrons scattering off the Hartree self-consistent potential, with the electron-density response also evaluated in the Hartree approximation, yielding $\rho_{\text {Har/Har }}$. A different way ${ }^{39}$ of inserting the local field factor corresponds to electrons scattering off the Hartree potential, with the electron-density response evaluated in LDA, yielding $\rho_{\mathrm{Har} / \mathrm{LDA}}$. Our results of Table IV, especially those for the large- $r_{s}$ metals, indicate that electrons scatter off the whole Kohn-Sham potential, including its exchange-correlation part as well as its Hartree part.

\section{BEYOND PERTURBATION THEORY: LATTICE CONSTANT AND BAND STRUCTURE}

We have seen that our density-based local pseudopotential, applied in second-order perturbation theory, yields a realistic description of the phonon frequencies and elastic constants of most simple metals. However, as we will show below, this does not mean that the pseudopotential is so weak that higher-order contributions to the energy are completely negligible. The correct interpretation ${ }^{11,44}$ is that these higher-order contributions have been to some extent folded into a low-order calculation by the perturbative method used to fix the parameters of the pseudopotential. The same observation presumably applies to the two-parameter local pseudopotential of Rasky and Milstein, ${ }^{45}$ which has been applied recently ${ }^{36,46}$ to calculate the pressure dependence of the elastic constants for the alkali metals.

In support of this position, Table $\mathrm{V}$ reports equilibrium lattice constants for bcc Na, hcp Mg, and fcc Al. Theoretical values were constructed by minimizing the total energies. To second order, the evanescent core pseudopotential reproduces the experimental values by construction. However, when these local pseudopotentials are applied nonperturbatively, the predicted lattice constants are too large by $1.6 \%$ for $\mathrm{Na}, 5.5 \%$ for $\mathrm{Mg}$, and $11.0 \%$ for $\mathrm{Al}$. Table $\mathrm{V}$ also shows that lattice constants predicted by the more realistic Hamann norm-conserving nonlocal pseudopotentials are too small by 


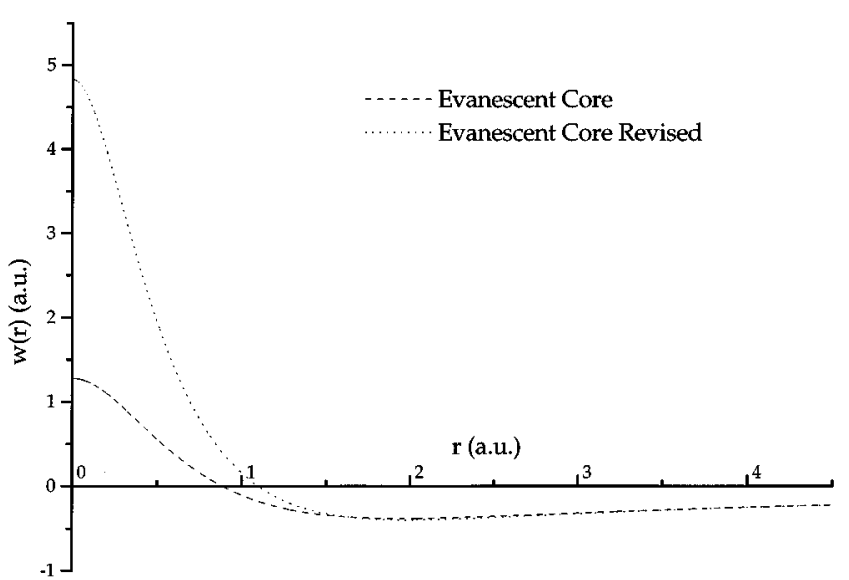

FIG. 1. Local evanescent core electron-ion pseudopotentials $w(r)$ for $\mathrm{Na}$. The two parameters of this potential have been fixed perturbatively in the dashed line, and nonperturbatively in the dotted line, to the experimental lattice constant and the all-electron interstitial density.

$6.0 \%$ for $\mathrm{Na}, 2.5 \%$ for $\mathrm{Mg}$, and $2.1 \%$ for $\mathrm{Al}$; this underestimation is principally due to the tendency of the local density approximation to exaggerate binding. All nonperturbative calculations were made within a plane-wave expansion, ${ }^{47}$ using a 40 Ry energy cutoff.

The two parameters of the local evanescent core pseudopotential may be revised for use in a nonperturbative calculation, as Nogueira et al. ${ }^{6}$ have done for Na. The revised potentials have a stronger and more short-ranged(harder) core repulsion, as Fig. 1 shows.

Only in a nonperturbative approach can one calculate a meaningful Kohn-Sham band structure. Figure 2 shows the band structure calculated for bcc $\mathrm{Na}$ (at the experimental lattice constant) using (a) the realistic nonlocal pseudopotential of Hamann, ${ }^{48}$ (b) our local evanescent core potential with revised (nonperturbative) parameters, and (c) the freeelectron model. We find that the band structure of $(b)$ is remarkably like that of (a), showing once again that a local pseudopotential fitted to the measured lattice constant and interstitial density is realistic.

The band structure of Fig. 2(b) is even closer to the band structure of the local pseudopotential seen by the $s$ electrons in the Hamann scheme, although this potential in real space (Fig. 1 of Ref. 6) bears little resemblance to the local pseudopotentials in our Fig. 1. Table VI presents numerical results for selected energy differences.

\section{CONCLUSIONS AND FUTURE DIRECTIONS}

In order to assess the strengths and weaknesses of our density-based local pseudopotential we have calculated phonon frequencies, elastic moduli, liquid metal resistivities, and the band structure, with results summarized in the abstract. Although the phonon frequencies and bulk moduli of $\mathrm{Ca}, \mathrm{Sr}$, and $\mathrm{Ba}$ are for the most part close to experiment, the spurious soft modes we find in these alkaline earths show that a nonlocal pseudopotential is needed to reflect the hybridization of low-lying $d$ states. For most of the other simple metals, we conclude that a local pseudopotential based on the interstitial and average valence electron density is a good
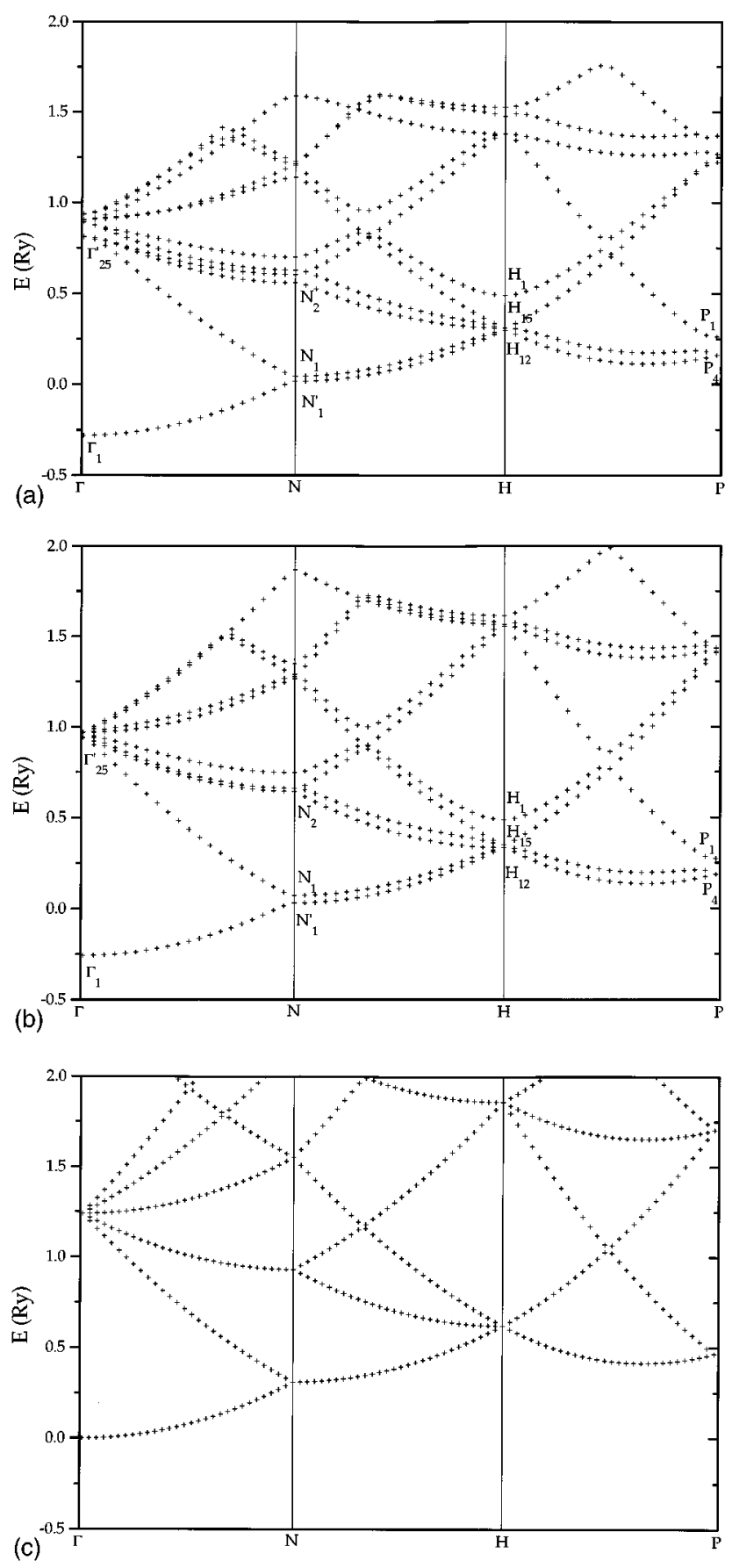

FIG. 2. Band structure for bcc Na, calculated using (a) Hamann's nonlocal pseudopotential, (b) our local evanescent core potential with revised (nonperturbative) parameters, and (c) the freeelectron model. The valence band and the first ten conduction bands are displayed.

choice which produces solid-state results in agreement with both experiment and nonlocal pseudopotential theory.

In all our work, we have used the local density approximation (LDA) for exchange and correlation. LDA is very accurate for the linear response of the density of a uniform electron gas, ${ }^{43}$ but less accurate in higher orders of perturbation theory. Thus we suggest that future nonperturbative calculations be based on the generalized gradient approximation. ${ }^{49,50}$ Such nonperturbative calculations are 
TABLE VI. Selected energy differences for the band structure of bcc sodium, in Ry. We compare our local evanescent core pseudopotential (with revised or nonperturbative parameters) against the nonlocal ( $s p d$ ) Hamann potential (Ref. 48) and its local $s$-electron part. All-electron augmented plane-wave (APW) results (Ref. 58) are also shown.

\begin{tabular}{lcccc}
\hline \hline Difference & $\begin{array}{c}\text { Local } \\
(\text { revised })\end{array}$ & $\begin{array}{c}\text { Hamann } \\
(s)\end{array}$ & $\begin{array}{c}\text { Hamann } \\
(s p d)\end{array}$ & $\begin{array}{c}\text { APW } \\
\text { (Ref. 58) }\end{array}$ \\
\hline$\Gamma_{25}^{\prime}-\Gamma_{1}$ & 1.195 & 1.179 & 1.090 & 1.062 \\
$N_{1}-N_{1}^{\prime}$ & 0.041 & 0.046 & 0.028 & 0.028 \\
$N_{2}-N_{1}$ & 0.571 & 0.559 & 0.515 & 0.504 \\
$H_{15}-H_{12}$ & 0.016 & 0.018 & 0.013 & 0.018 \\
$P_{1}-P_{4}$ & 0.086 & 0.101 & 0.102 & 0.098 \\
\hline \hline
\end{tabular}

needed for the description of open crystal structures like that of silicon or diamond.

Local pseudopotentials cannot be as realistic as nonlocal ones, but still have a role to play in an age of powerful computers. First, for the description of disordered condensed phases such as alloys and liquid metals, both locality and suitability to low-order perturbation theory have key computational advantages. Second, the simplicity of local pseudopotentials invites the discovery or explanation of chemical trends among the $s p$-bonded elements. We are currently searching for a unified understanding of equilibrium crystal structures at normal and compressed volumes, and more generally for a universal local pseudopotential controlled by only two inputs, the chemical valence and the average valence-electron density. Third, local pseudopotentials define quasirealistic systems on which density-functional approximations for the exchange-correlation energy can be tested against nearly exact calculations that often yield richer and more precise information than experiments. For example, the static linear-response function of the uniform electron gas is known exactly at the exchange-only level, ${ }^{51}$ and almost exactly when correlation is included. ${ }^{43}$ Beyond linear response, quantum Monte Carlo (QMC) simulations ${ }^{52}$ produce a nearly exact result for systems of interacting electrons by evaluating only a representative random sampling of the wave function. QMC typically requires a pseudopotential and works far more efficiently when that pseudopotential is local.

\section{ACKNOWLEDGMENTS}

We thank M. Scheffler for kindly providing the computer program FHI94MD, and J. A. Paixão, R. Taylor, and J. H. Rose for helpful discussion or information. This work has been supported by the U.S. National Science Foundation under Grant No. DMR95-21353, by the Louisiana Educational Quality Support Fund, and by the Portuguese Praxis XXI program (PRAXIS/2/2.1/FIS/26/94).
${ }^{1}$ W. A. Harrison, Pseudopotentials in the Theory of Metals (Benjamin, New York, 1967).

${ }^{2}$ J. Hafner, From Hamiltonians to Phase Diagrams (SpringerVerlag, Berlin, 1987).

${ }^{3}$ W. A. Harrison, Electronic Structure and the Properties of Solids (Dover, New York, 1980).

${ }^{4}$ C. Fiolhais, J. P. Perdew, S. Q. Armster, J. M. MacLaren, and M. Brajczewska, Phys. Rev. B 51, 14001 (1995); 53, 13193 (E) (1996).

${ }^{5}$ D. R. Hamann, M. Schlüter, and C. Chiang, Phys. Rev. Lett. 43, 1494 (1979).

${ }^{6}$ F. Nogueira, C. Fiolhais, J. He, J. P. Perdew, and A. Rubio, J. Phys. Condens. Matter 8, 287 (1996).

${ }^{7}$ J. H. Rose and H. B. Shore, Phys. Rev. B 49, 11588 (1994).

${ }^{8}$ J. A. Moriarty, Phys. Rev. B 26, 1754 (1982).

${ }^{9}$ L. Dagens, M. Rasolt, and R. Taylor, Phys. Rev. B 11, 2726 (1975).

${ }^{10}$ M. Rasolt and R. Taylor, Phys. Rev. B 11, 2717 (1975).

${ }^{11}$ J. P. Perdew and S. H. Vosko, J. Phys. F 6, 1421 (1976).

${ }^{12}$ G. J. Vázquez and L. F. Magaña, J. Phys. (France) 49, 497 (1988).

${ }^{13}$ M. Manninen, P. Jena, R. M. Nieminen, and J. K. Lee, Phys. Rev. B 24, 7057 (1981).

${ }^{14}$ G. J. Vázquez and L. F. Magaña, Phys. Lett. 122, 267 (1987).

${ }^{15}$ L. F. Magaña and G. J. Vázquez, J. Phys. Condens. Matter 7, L393 (1995).

${ }^{16}$ D. D. Ling and C. D. Gelatt, Phys. Rev. B 22, 557 (1980).

${ }^{17}$ D. Sen, S. K. Sarkar, S. Sengupta, and D. Roy, Phys. Status Solidi B 115, 593 (1983)

${ }^{18}$ L. D. Landau and E. M. Lifshitz, Theory of Elasticity, 3rd Edi- tion, Course of Theoretical Physics (Butterworth-Heinemann, Oxford, 1986).

${ }^{19}$ A. Cottrell, Introduction to the Modern Theory of Metals (The Universities Press, Belfast, 1988).

${ }^{20}$ E. G. Brovman, Y. Kagan, and A. Kholas, Sov. Phys. JETP 34, 394 (1972).

${ }^{21}$ K. A. Gschneidner, Jr., in Solid State Physics: Advances in Research and Applications, edited by F. Seitz and D. Turnbull (Academic, New York, 1964), Vol. 16, p. 275.

${ }^{22}$ F. Milstein, J. Appl. Phys. 44, 3825 (1973).

${ }^{23}$ C. Kittel, Introduction to Solid State Physics (Wiley, New York, 1971).

${ }^{24}$ M. Born and K. Huang, Dynamical Theory of Crystal Lattices (Clarendon, Oxford, 1954).

${ }^{25}$ D. C. Wallace, Phys. Rev. 182, 778 (1969).

${ }^{26}$ C. J. Pethick, Phys. Rev. B 2, 1789 (1970).

${ }^{27}$ W. Jones, J. Phys. C 6, 2833 (1973).

${ }^{28}$ S. H. Taole and H. R. Glyde, Can. J. Phys. 57, 1870 (1979).

${ }^{29}$ R. Pynn, Phys. Rev. B 5, 4826 (1972).

${ }^{30}$ E. Wigner and F. Seitz, Phys. Rev. 43, 804 (1933).

${ }^{31}$ E. Wigner and F. Seitz, Phys. Rev. 46, 509 (1934).

${ }^{32}$ T. Kraft, P. M. Marcus, M. Methfessel, and M. Scheffler, Phys. Rev. B 48, 5886 (1993).

${ }^{33}$ V. L. Sliwko, P. Mohn, K. Schwarz, and P. Blaha, J. Phys. Condens. Matter 8, 799 (1996).

${ }^{34}$ J. A. Moriarty, Phys. Rev. B 34, 6738 (1986).

${ }^{35}$ D. A. Young, Phase Diagrams of the Elements (University of California Press, Berkeley, 1991).

${ }^{36}$ F. Milstein and D. J. Rasky, Phys. Rev. B 54, 7016 (1996).

${ }^{37}$ J. M. Ziman, Philos. Mag. 6, 1013 (1961). 
${ }^{38}$ N. W. Ashcroft and J. Lekner, Phys. Rev. 145, 83 (1966).

${ }^{39}$ A. P. Horsfield and N. W. Ashcroft, J. Phys. Condens. Matter 5, 3925 (1993).

${ }^{40}$ C. Rottman and J. B. Van Zytfeld, J. Phys. F 9, 2049 (1979).

${ }^{41}$ A. E. Sharp and P. V. Smith, Solid State Commun. 15, 383 (1974).

${ }^{42}$ V. Heine, P. Nozières, and J. W. Wilkins, Philos. Mag. 13, 741 (1966).

${ }^{43}$ S. Moroni, D. M. Ceperley, and G. Senatore, Phys. Rev. Lett. 75, 689 (1995).

${ }^{44}$ S. Benckert, Phys. Status Solidi B 69, 483 (1975).

${ }^{45}$ D. J. Rasky and F. Milstein, Phys. Rev. B 33, 2765 (1986).

${ }^{46}$ F. Milstein, H. E. Fang, X.-Y. Gong, and D. J. Rasky, Solid State Commun. 99, 807 (1996).

${ }^{47}$ R. Stumpf and M. Scheffler, Comput. Phys. Commun. 79, 447 (1994).

${ }^{48}$ D. R. Hamann, Phys. Rev. B 40, 2980 (1989).

${ }^{49}$ J. P. Perdew, K. Burke, and M. Ernzerhof, Phys. Rev. Lett. 77, 3865 (1996).
${ }^{50}$ D. R. Hamann, Phys. Rev. Lett. 76, 660 (1996).

${ }^{51}$ P. A. Antoniewicz and L. Kleinman, Phys. Rev. B 31, 6779 (1985).

${ }^{52}$ R. M. Martin and V. D. Natoli, in Computational Approaches to Novel Condensed Matter Systems, edited by D. Neilson and M. P. Das (Plenum, New York, 1995), p. 7.

${ }^{53}$ J. Mizuki and C. Stassis, Phys. Rev. B 34, 5890 (1986).

${ }^{54}$ J. Mizuki, Y. Chen, K.-M. Ho, and C. Stassis, Phys. Rev. B 32, 666 (1985).

${ }^{55}$ C. Stassis, J. Zaretsky, D. K. Misemer, H. L. Skriver, B. N. Harmon, and R. M. Nicklow, Phys. Rev. B 27, 3303 (1983).

${ }^{56}$ U. Buchenau, M. Heiroth, H. R. Schober, J. Evers, and G. Oehlinger, Phys. Rev. B 30, 3502 (1984).

${ }^{57}$ G. Simmons and H. Wang, Single Crystal Elastic Constants and Calculated Aggregate Properties (MIT, Cambridge, MA, 1971).

${ }^{58}$ D. A. Papaconstantopoulos, Handbook of the Band Structure of Elemental Solids (Plenum, New York, 1986). 